\title{
Upregulation of ZBTB7A exhibits a tumor suppressive role in gastric cancer cells
}

\author{
GUANG SUN, BO PENG, QUAN XIE, JIANWEN RUAN and XIANWEN LIANG
}

\begin{abstract}
Department of Gastroenterology Surgery, Haikou Municipal People's Hospital, Central South University Xiangya School of Medicine Affiliated Haikou Hospital, Haikou, Hainan 570208, P.R. China
\end{abstract}

Received December 16, 2016; Accepted July 25, 2017

DOI: $10.3892 / \mathrm{mmr} .2017 .8104$

\begin{abstract}
Gastric cancer presents as a complex solid tumor and is the third leading cause of global cancer-associated mortality. The genetic alterations in gastric cancer remain unclear and deserve further investigation. Mining The Cancer Genome Atlas gastric adenocarcinoma dataset identified a frequent loss of the zinc finger and BTB domain containing 7A (ZBTB7A) gene locus and a significant correlation between low ZBTB7A expression and poor patient survival. ZBTB7A belongs to the POZ/BTB and Kruppel transcription factor family. In the present study, overexpression of ZBTB7A in a gastric cancer cell line induced cell cycle arrest at the $S$ phase. Upregulation of ZBTB7A also promoted apoptosis and repressed cell migration. The results of the present study indicated that ZBTB7A functions as a tumor suppressor in gastric cancer cells. Understanding the role of ZBTB7A in gastric cancer may provide important clinical insight for treatment.
\end{abstract}

\section{Introduction}

Gastric cancer (GC) is the fifth most common type of cancer worldwide and the third leading cause of global cancer-associated mortality (1). GC is a complex solid tumor arising from genetic alterations, environmental interactions and host-associated factors (2). GC is a major contributor to the worldwide disability-adjusted life-years among patients with cancer (3). Achieving greater understanding of the molecular genomic mutations in GC is pivotal to improving therapies and outcomes for patients with GC. Recently great progress has been achieved in GC, including the identification of novel cellular pathways and molecular components (4). The Cancer Genome Atlas (TCGA) project recently classified GC

Correspondence to: Dr Guang Sun, Department of Gastroenterology Surgery, Haikou Municipal People's Hospital, Central South University Xiangya School of Medicine Affiliated Haikou Hospital, 43 Renmin Road, Haikou, Hainan 570208, P.R. China

E-mail: sunguang1976@126.com

Key words: gastric cancer, zinc finger and BTB domain containing 7A, apoptosis, migration, cell cycle as possessing four genomic subtypes based on $\sim 300$ molecular profiles of patients with GC (5). Further study of the molecular mechanisms and cellular pathways of GC may provide novel insight for the improvement of early diagnostic techniques, precision therapies and prognostic predictions for patients with GC.

The gene, zinc finger and BTB domain containing 7A (ZBTB7A) is also known as lymphoma related factor (6), factor that binds to inducer of short transcripts of human immunodeficiency virus type 1 (7) and osteoclast-derived zinc finger (8). ZBTB7A is one member of the protection of telomeres protein POZ-1/BTB and Kruppel (POK) transcription factors family (9-11). The POK transcription factor family has been demonstrated to bind DNA via a Kruppel-like DNA-binding domain and represses transcriptional activity by recruiting co-repressor complexes via the POZ domain (12). ZBTB7A was reported to promote oncogenesis through its capacity to repress the transcription of an important tumor suppressor gene alternative reading frame (ARF) (11). Previously, aberrant ZBTB7A overexpression has been reported in a number of different types of human cancer, including breast cancer, non-small cell lung cancer (NSCL), lymphoma and ovarian cancer (11,13-17), suggesting that ZBTB7A acts as novel proto-oncogene in multiple tissues.

However, the frequent chromosomal deletion of the ZBTB7A gene locus (19p13.3) in multiple types of human cancer (18-20) suggests it is not a proto-oncogene. This evidence implies that the function of ZBTB7A is determined by its context in solid tumors. A previous study demonstrated that the loss of ZBTB7A promoted progression of mouse prostate cancer by activating transcription factor SOX9-dependent signaling pathway in a phosphatidylinositol 3,4,5-trisphosphate 3-phosphatase and dual-specificity protein phosphatase PTEN (PTEN)-loss background (21). Another study also reported that ZBTB7A acts as a transcriptional repressor by binding directly to the promoter of glycolytic genes and repressing their transcription (22). Liu et al (20) also demonstrated that ZBTB7A can bind directly to the promoter region of the melanoma cell adhesion molecule to suppress its transcription and represses melanoma metastasis. These reports suggest that ZBTB7A can act as a tumor suppressor under certain circumstances. Whether ZBTB7A acts as oncogene or tumor suppressor is context-dependent in different types of cancer. 
Frequent deletions in the ZBTB7A gene locus have been reported in a number of different types of cancer (18-20). In the present study, it was hypothesized that ZBTB7A may function as a tumor suppressor in GC. Recently, one study demonstrated that downregulation of ZBTB7A by small interfering (si)RNA suppressed the migratory ability of GC cells without an impact on cell proliferation and apoptosis (23). However, it remains unclear whether overexpression of ZBTB7A in a GC cell line will affect cell proliferation, apoptosis and migratory capacity. Therefore, it is necessary to further investigate the function and potential mechanism of ZBTB7A in GC, which may be a novel target for treatment and improve clinical outcome.

\section{Materials and methods}

Human cancer genomic analysis. Copy number alterations (CNA) and mRNA data for the ZBTB7A and PTEN genes in 441 cases of human gastric adenocarcinoma were downloaded from TCGA database $(24,25)$. Z-score indicates ZBTB7A mRNA expression levels. For the analysis of overall patient survival, the ZBTB7A expression data, along with the survival data, were divided into two groups, 'ZBTB7A low expression' and 'ZBTB7A high expression' based on the median expression level of ZBTB7A.

Cell culture. The gastric adenocarcinoma cell line SGC-7901 was bought from the Type Culture Collection of the Chinese Academy of Sciences, (Shanghai, China). SGC-7901 cells were cultured in RPMI-1640 medium (cat no. 10-013-CVR; Corning Incorporated, Corning, NY, USA) with $10 \%$ fetal bovine serum (FBS, cat no. VS500T; Ausbian, Vian-Saga Company, Shanghai, China; http://www.viansaga.com/h-pd-1. html\#_pp=2_731) and 1\% penicillin/streptomycin. 293 cells (cat no. R79007; Thermo Fisher Scientific, Inc., Waltham, MA, USA) were cultured in DMEM (cat no. R10-017-CVR; Corning Incorporated) with $10 \%$ FBS. All cells were cultured at $37^{\circ} \mathrm{C}$ in a humidified incubator (MCO-15A; Sanyo, Osaka, Japan) containing $5 \% \mathrm{CO}_{2}$.

$R N A$ isolation and reverse transcription-quantitative polymerase chain reaction ( $R T-q P C R)$. Total RNA was isolated with the SuperfecTRI ${ }^{\mathrm{TM}}$ reagent (cat no. 3101-100; Shanghai Pufei Biotech Co., Ltd., Shanghai, China). A total of $1 \mu \mathrm{g}$ RNA was reverse transcribed into cDNA using the Moloney-murine leukemia virus kit (cat no. M1705; Promega Corporation, Madison, WI, USA). qPCR was performed in a Real-Time PCR machine system (cat no. MX3000p; Agilent Technologies, Inc., Santa Clara, CA, USA), using cDNA and SYBR Master mixture (cat no. DRR041B; Takara Biotechnology Co., Ltd., Dalian, China) (26-27). The following cycling conditions were used: One cycle for $30 \mathrm{sec}$ at $95^{\circ} \mathrm{C}, 40$ cycles for $5 \mathrm{sec}$ at $95^{\circ} \mathrm{C}$ and $30 \mathrm{sec}$ at $60^{\circ} \mathrm{C}$, then one cycle of dissociation including $15 \mathrm{sec}$ at $95^{\circ} \mathrm{C}, 30 \mathrm{sec}$ at $60^{\circ} \mathrm{C}$ and $15 \mathrm{sec}$ at $95^{\circ} \mathrm{C}$. GAPDH was used as an endogenous control. The primer sequences of GAPDH and ZBTB7A genes were as follows: GAPDH forward, 5'-TGACTTCAACAGCGACACCCA-3' and reverse; 5'-CACCCTGTTGCTGTAGCCAAA-3'. ZBTB7A forward, 5'-CATCTGCGAGAAGGTCATCC-3' and reverse 5'-TGT CCTGCCTGGTGAAGC-3' $(26,28)$.
Plasmid construction and lentiviral transfection. The pGV115-ZBTB7A-FLAG-green fluorescent protein(GFP)-puro plasmid (20 $\mu \mathrm{g}$; Shanghai GeneChem Co., Ltd., Shanghai, China) was constructed by inserting a full-length human cDNA of ZBTB7A-FLAG gene into a pGV115-GFP-puro plasmid vector. The pGV115-ZBTB7A-FLAG-GFP-puro plasmid along with another two lentiviral packaging plasmids pHelper1.0 and pHelper2.0 (15 $\mu \mathrm{g}$ each; both from Shanghai GeneChem Co., Ltd.) was cotransfected into 293 cells using Lipofectamine ${ }^{\mathrm{TM}}$ reagent (Invitrogen; Thermo Fisher Scientific, Inc.). The lentiviral supernatant was collected, concentrated and purified in the 48-72 $\mathrm{h}$ following cotransfection. The SGC-7901 cell line was treated with an equal concentration of $2 \times 10^{8}(\mathrm{PFU} / \mathrm{ml})$ of lentiviral supernatant for transfection. Cells were observed for GFP expression under a fluorescence microscope after $72 \mathrm{~h}$ viral transfection.

Western blotting. Protein was extracted using $2 \mathrm{X}$ radioimmunoprecipitation assay lysis buffer (cat no. WB-0071; Dingguo Bio Co., Ltd, Shanghai, China) from whole cells. The protein concentration was measured using a bicinchoninic acid protein assay kit (cat no. P0010S). The cell lysate was separated using 10\% SDS-PAGE with loading $30 \mu \mathrm{g}$ protein and then transferred onto a polyvinylidene difluoride membrane (cat no. IPVH00010; EMD Millipore, Billerica, MA, USA) at $4^{\circ} \mathrm{C}$ and $300 \mathrm{~mA}$ for $150 \mathrm{~min}$ and blotted with $5 \%$ milk in $1 \mathrm{X}$ TBST buffer at room temperature for $1 \mathrm{~h}$. Membranes were then blotted with diluted primary antibodies at $4^{\circ} \mathrm{C}$ overnight for GAPDH (1:5,000; cat no. SC-32233; Santa Cruz Biotechnology, Inc., Dallas, TX, USA), FLAG (1:3,000; cat no. F1804; Sigma-Aldrich; Merck KGaA, Darmstadt, Germany) and ZBTB7A (1:2,000; cat no. Ab175918; Abcam, Cambridge, UK). Survivin-3FLAG-GFP was used as FLAG positive control. A goat-anti-rabbit secondary antibody (1:5,000; cat no. sc-2004; Santa Cruz Biotechnology, Inc.) were then incubated at room temperature for $1.5 \mathrm{~h}$. Subsequently, Pierce $^{\mathrm{TM}}$ ECL western blotting substrate was added for exposure (Thermo Fisher Scientific, Inc.). Each western blot analysis was performed at least three times independently.

MTT assay. A total of 2,000 healthy cells/well were seeded into a 96-well plate (cat no. 3599; Corning Incorporated) with $100 \mu \mathrm{l}$ medium. A total of $20 \mu \mathrm{l} 5 \mathrm{mg} / \mathrm{ml}$ MTT reagent (cat no. JT343; Genview, Beijing, China) was added to each well $\sim 4 \mathrm{~h}$ prior to detection. Next, the culture medium was removed and $100 \mu \mathrm{l} /$ well dimethyl sulfoxide was added. Following $5 \mathrm{~min}$ incubation, the optical density of the cells was analyzed at 490/570 $\mathrm{nm}$ emission/absorption wavelength on a Tecan infinite machine (cat no. M2009PR; Tecan Group, Ltd., Mannedorf, Switzerland).

Cell cycle assay. Cells were seeded into 6-cm dishes with $4 \mathrm{ml}$ medium following lentiviral transfection. Then cells were collected after 3 days. Transfected cells were trypsinized, washed, fixed for $1 \mathrm{~h}$ at $4^{\circ} \mathrm{C}$ with $75 \%$ ethanol and incubated with propidium iodide (PI) dye (cat no. P4170; Sigma-Aldrich; Merck $\mathrm{KGaA}$ ) for $30 \mathrm{~min}$ at room temperature. Stained cells were measured for cell cycle phase distribution using a flow cytometer (Guava ${ }^{\circledR}$ easyCyte HT; EMD Millipore). Cell cycle data was analyzed using FlowJo software (version 7.6.1; 
A
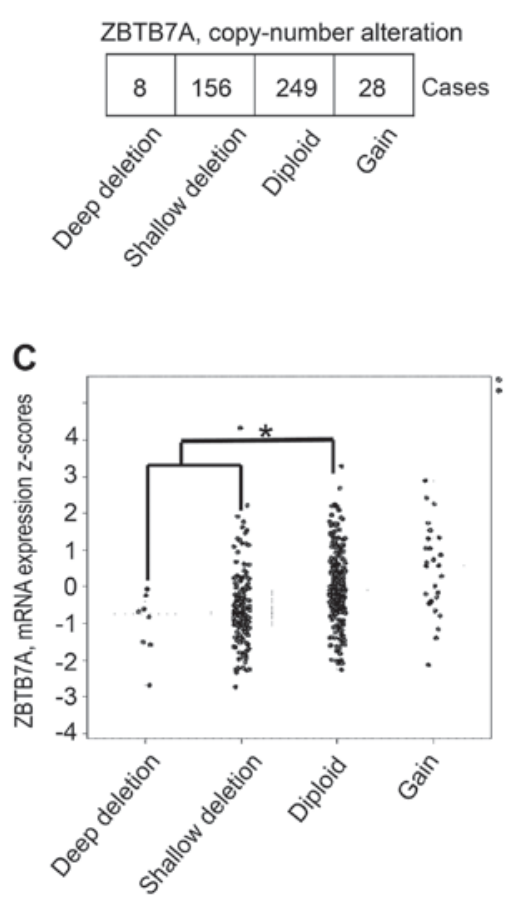

B

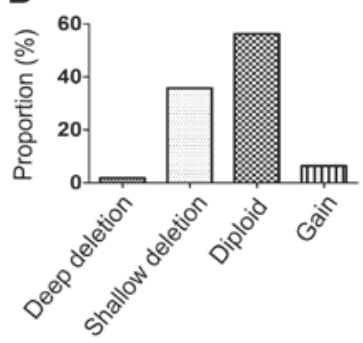

D

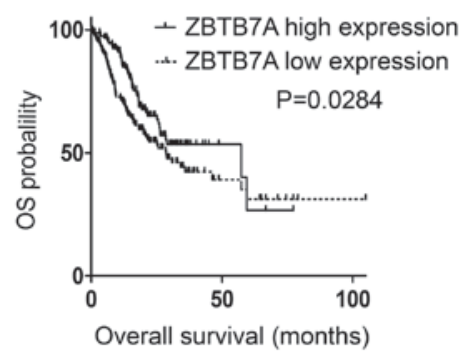

Figure 1. ZBTB7A CNA, mRNA distribution and overall survival data among human gastric adenocarcinoma cases (provisional, 441 cases) from TCGA dataset. Deep deletion refers to a homozygous allele deletion; shallow deletion refers to a hemizygous allele deletion; diploid refers to two normal alleles; gain refers to >two alleles. (A) Distribution of ZBTB7A CNA of 441 patients in TCGA. (B) ZBTB7A CNA percentage distribution (C) ZBTB7A mRNA of two ZBTB7A deletion groups together was significantly decreased compared with the diploid group. $\mathrm{P}=0.012$. Z-score indicates ZBTB7A mRNA expression level. (D) The association between ZBTB7A expression and patient overall survival. Log-rank test: $\mathrm{P}=0.0284$ and * $\mathrm{P}<0.05$. CNA, copy number alteration; TCGA, The Cancer Genome Atlas; OS, overall survival; ZBTB7A, zinc finger and BTB domain containing 7A.

FlowJo LLC, Ashland, OR, USA). The cell cycle assay was repeated three times independently.

Apoptosis assay. Cells were seeded into 6-well plates with $2 \mathrm{ml}$ medium following transfection and were harvested 2 days later. Cells were stained using the Annexin V-APC\&PI Apoptosis Detection kit (cat no. 88-8007; eBioscience; Thermo Fisher Scientific, Inc.), according to the manufacturer's protocol. Cells were stained to measure apoptosis using flow cytometry software (version 7.6.1; FlowJo LLC). The apoptosis assay was repeated three times independently.

Scratch assay. An equal number of $3 \times 10^{4}$ cells/well were seeded into a 96-well plate following transfection. The cell monolayer was scratched in a straight line in each well. The line was marked and images were captured under phase-contrast microscope (Zeiss; XDS-100). Cells were cultured for 8 and $24 \mathrm{~h}$. Following incubation, images were retaken in the same region centered on the line. The width was measured and recorded at 0,8 and $24 \mathrm{~h}$. The migratory rate was calculated as [(width at $0 \mathrm{~h}$ - width at 8 or $24 \mathrm{~h}$ )/width at $0 \mathrm{~h}$ ]. The rate of migration was analyzed. The scratch assay was repeated four times independently.

Statistical analysis. All results were analyzed using GraphPad Prism software (version 5; GraphPad software, Inc., La Jolla, CA, USA) and data were presented as the mean \pm standard error of the mean. The data were analyzed using a Student's t-test for comparisons between two groups. Multiple comparison tests were performed using two-way analysis of variance (ANOVA) and Bonferroni post-hoc tests to analyze the data from the cell cycle distribution and migration assays. One-way ANOVA was used to analyze the data from the apoptosis data, followed by Tukey's test. Log-rank test and Kaplan-Meier estimators were performed. $\mathrm{P}<0.05$ was considered to indicate a statistically significant difference.

\section{Results}

Frequent loss of ZBTB7A and its association with patient overall survival in the human gastric adenocarcinoma database. As frequent chromosomal deletions at the ZBTB7A gene locus have been reported in a number of different types of human cancer (18-20), in the present study the ZBTB7A gene was investigated in human gastric adenocarcinoma. CNA, mRNA expression and overall survival data from 441 patients were downloaded from the TCGA provisional dataset. A total of 8 patients $(1.8 \%)$ presented with a homozygous deletion of ZBTB7A and 156 patients (35.37\%) exhibited hemizygous deletions (Fig. 1A and B). A total of $37.17 \%$ of patients with gastric adenocarcinoma exhibited a ZBTB7A gene deletion, with 56.56\% exhibiting no deletion (Fig. 1B). The ZBTB7A mRNA expression level of the two gene deletion groups was significantly decreased compared with the normal diploid group (Fig. 1C). The overall survival data were analyzed and it was demonstrated that the ZBTB7A high expression group exhibited a median survival of 57.39 months, which was significantly increased compared with 28.71 months in the 
A

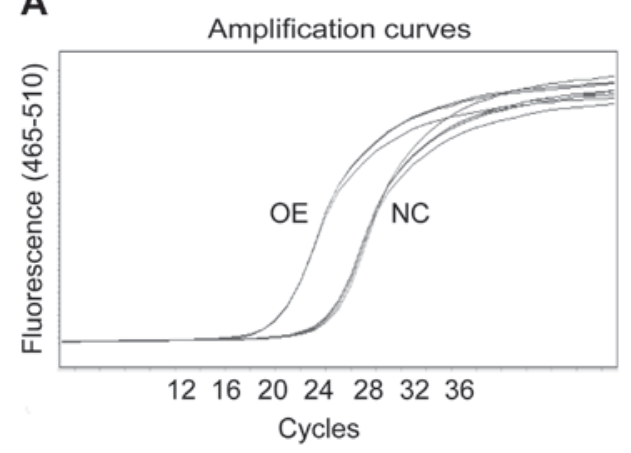

B

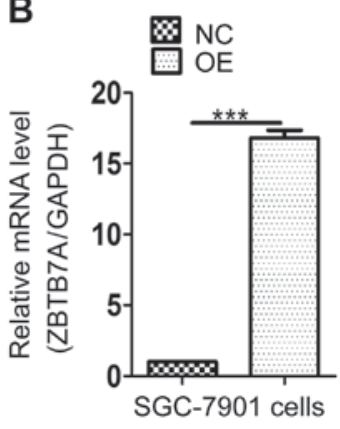

C SGC-7901 cells

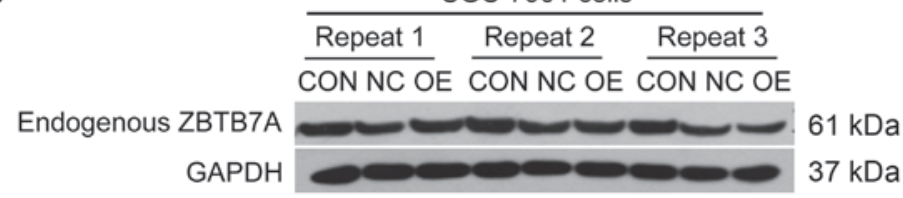

D

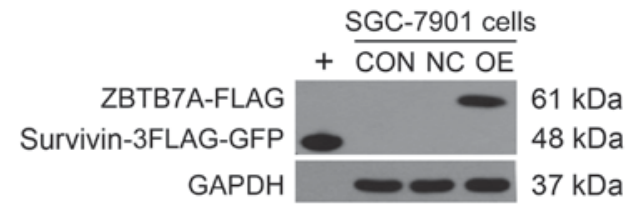

Figure 2. Establishment of ZBTB7A overexpression SGC-7901 cell line. (A) Reverse transcription-quantitative polymerase chain reaction amplification curves of NC and OE group. (B) The relative mRNA expression level of ZBTB7A/GAPDH in NC and OE group. (C) Western blotting demonstrated the endogenous ZBTB7A protein level of CON, NC and OE group in SGC-7901 cell line. (D) Ectopic ZBTB7A-FLAG protein was only detected in OE group. GAPDH was used as loading control. Survivin-3FLAG-GFP served as positive control as ' + .' .*** $\mathrm{P}<0.001$. NC, negative control; CON, control; OE, overexpression; GFP, green fluorescent protein; ZBTB7A, zinc finger and BTB domain containing 7A.

ZBTB7A low expression group (Fig. 1D). These results implied that low expression of ZBTB7A was associated with a poor median survival. The data indicated that ZBTB7A may function as potential tumor suppressor in gastric adenocarcinoma. To further investigate this, a gain-of-function experiment was performed for ZBTB7A, and its impact on cell proliferation, apoptosis and migration in the GC cell line SGC-7901 was investigated.

Establishment of a ZBTB7A overexpression system in the SGC-7901 cell line. To produce a cell line that overexpressed ZBTB7A, SGC-7901 cells were transfected using a lentiviral vector. GFP expression by the negative control (NC) and overexpression (OE) group confirmed that transfection was successful (data not shown). The amplification curve of the RT-qPCR results confirmed that the mRNA level of the OE group reached a peak more rapidly than the $\mathrm{NC}$ group (Fig. 2A). The ZBTB7A/GAPDH mRNA expression analysis demonstrated that the level of mRNA in the OE group was $\sim 16$ times that of the NC group (Fig. 2B), suggesting that ZBTB7A mRNA was successfully over-expressed. In the western blot analysis, endogenous ZBTB7A expression was equal in the control (CON), $\mathrm{NC}$ and $\mathrm{OE}$ groups (Fig. 2C). Ectopic ZBTB7A-FLAG protein was successfully expressed only in OE group with Survivin-3FLAG-GFP serving as positive control (Fig. 2D). The data indicated that the ZBTB7A overexpression cell line was successfully constructed. These cells were used for further assays.
Ectopic ZBTB7A expression results in cell cycle inhibition at $S$ phase. To investigate whether gain-of-function of ZBTB7A affects GC cell proliferation or the cell cycle, a cell cycle and MTT assay were used. In the MTT assay, no significant difference in proliferation between the $\mathrm{CON}, \mathrm{NC}$ and $\mathrm{OE}$ groups was detected (data not shown), which suggested that overexpression of ZBTB7A may not affect cell proliferation. An increased proportion of cells were in the $\mathrm{S}$ phase in the $\mathrm{OE}$ group compared with the $\mathrm{CON}$ or $\mathrm{NC}$ groups according to the cell cycle assay (Fig. 3A). Statistical analyses were performed following three repeats. A decreased percentage of cells in OE group were demonstrated to be in the G1 phase compared with the $\mathrm{CON}(\mathrm{P}=0.0007)$ and $\mathrm{NC}(\mathrm{P}=0.0021)$ groups, with no difference between the $\mathrm{CON}$ and $\mathrm{NC}$ groups (Fig. 3B). The $\mathrm{P}$-values for the $\mathrm{S}$ phase in the $\mathrm{OE}$ group were $\mathrm{CON}(\mathrm{P}=0.0015)$ and $\mathrm{NC}(\mathrm{P}=0.0022)$, with no difference between $\mathrm{CON}$ and $\mathrm{NC}$ groups (Fig. 3B). No difference was detected in the percentage of cells in the G2 phase between the three groups (Fig. 3B). Overexpression of ZBTB7A in the SGC-7901 cell line induced an abnormal number of cells to arrest in the $\mathrm{S}$ phase of the cell cycle but without significant impact on cell proliferation.

Gain-of-function of ZBTB7A in SGC-7901 cell line induces apoptosis. To further investigate the impact on cell death or apoptosis of overexpressing ZBTB7A, an apoptosis assay was used. In the NC group, there were $\sim 90.8 \%$ GFP-positive cells and $92.78 \%$ GFP-positive cells in the OE group were detected, with only $0.9 \%$ GFP-positive cells in CON group (Fig. 4A). 


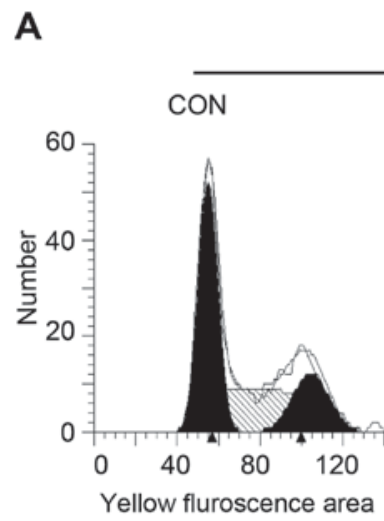

SGC-7901 cells
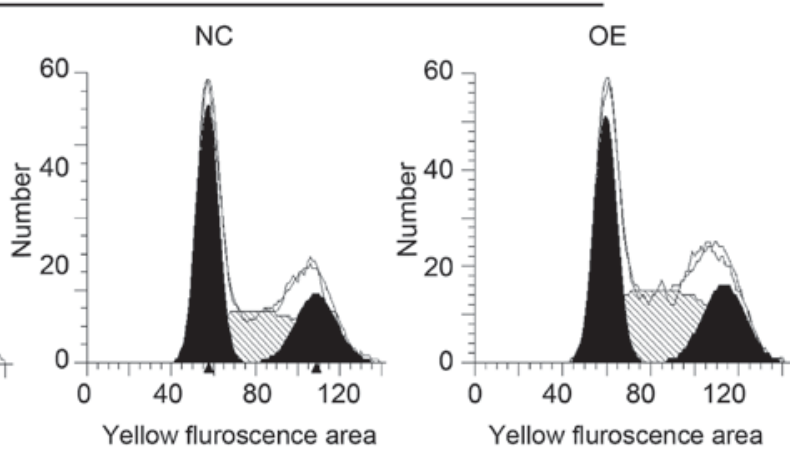

B

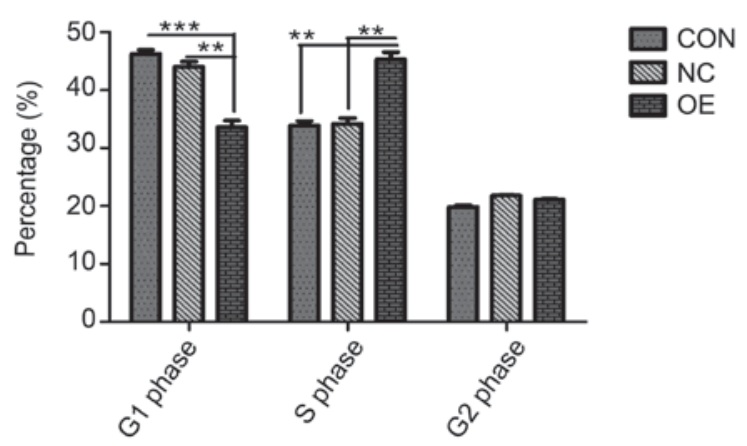

Figure 3. Overexpression of ZBTB7A induces cell cycle inhibition at the S stage. (A) The distribution of cells in different phases of the cell cycle in the CON $\mathrm{NC}$ and $\mathrm{OE}$ groups, measured by yellow fluorescence in a flow cytometry machine. (B) Statistical analysis of percentage of cells in the G1, S and G2 phases. ${ }^{*} \mathrm{P}<0.05,{ }^{* *} \mathrm{P}<0.01,{ }^{* * *} \mathrm{P}<0.001$. NC, negative control; CON, control; OE, overexpression; ZBTB7A, zinc finger and BTB domain containing 7A.

\section{A} SGC-7901 cells

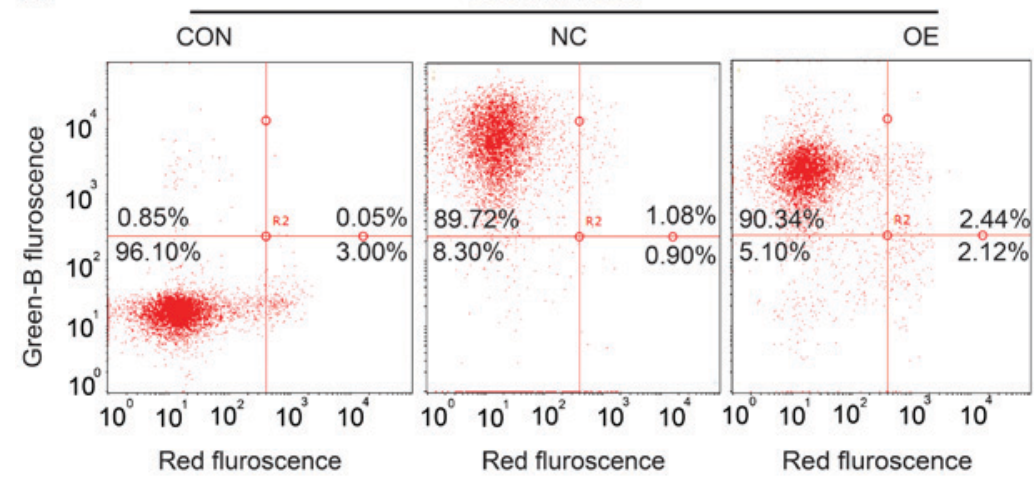

B

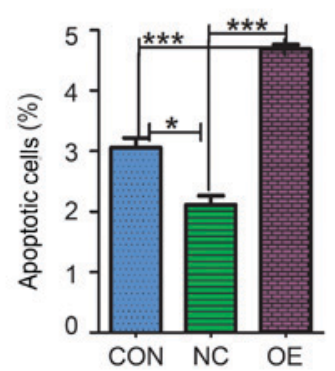

Figure 4. Apoptosis assay demonstrates that ZBTB7A overexpression in SGC-7901 cell line promotes apoptosis. (A) The distribution of stained cells by yellow fluorescence in flow cytometry machine. (B) Statistical analysis of the percentage of apoptotic cells in the CON, NC and OE groups. ${ }^{*} \mathrm{P}<0.05,{ }^{* * * *} \mathrm{P}<0.001 . \mathrm{NC}$, negative control; CON, control; OE, overexpression; ZBTB7A, zinc finger and BTB domain containing 7A.

The apoptosis percentage of CON, NC and OE groups was $3.06 \pm 0.27,2.11 \pm 0.26$ and $4.69 \pm 0.12 \%$, respectively (Fig. $4 \mathrm{~B}$ ).
Statistical analysis identified that percentage of apoptotic cells in the OE group was significantly increased compared with the 
A

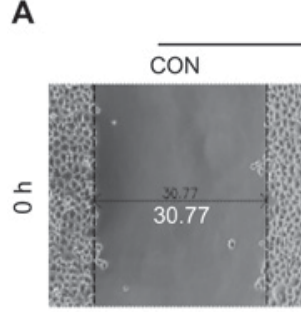

SGC-7901 cells
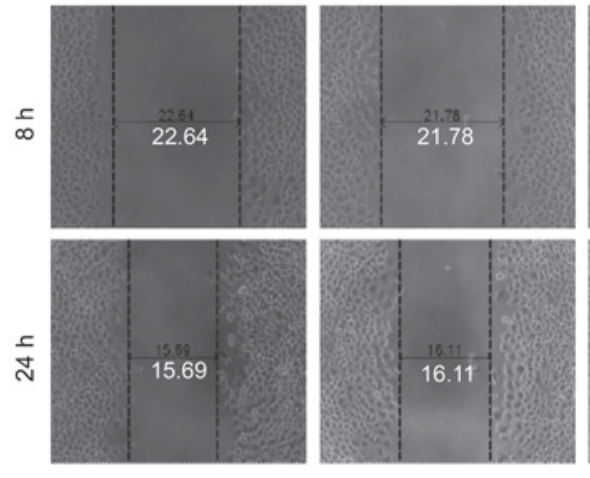

B

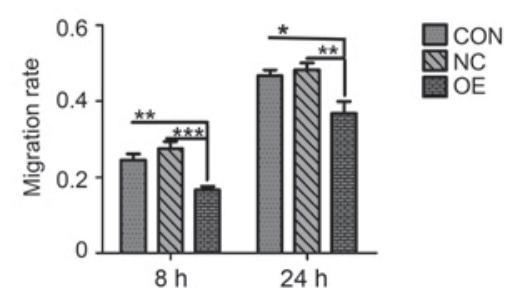

Figure 5. Scratch assay demonstrates that ZBTB7A suppresses cell migratory capacity. (A) Images were captured under 100x magnification using a bright field microscope at 0,8 and $24 \mathrm{~h}$ following a scratch assays being performed. Distances were measured, recorded and marked. (B) Migration rates of each group at different time points were compared and analyzed. ${ }^{*} \mathrm{P}<0.05,{ }^{* *} \mathrm{P}<0.01,{ }^{* * *} \mathrm{P}<0.001$. NC, negative control; CON, control; OE, over-expression; ZBTB7A, zinc finger and BTB domain containing 7A.

CON group $(\mathrm{P}=0.0007)$ and $\mathrm{NC}$ group $(\mathrm{P}=0.0001)$. These data indicated that gain-of-function of ZBTB7A in SGC-7901 cell promoted cell apoptosis.

Upregulation of ZBTB7A suppresses cell migration. The migratory and invasive ability is another key characteristic of GC cells, which facilitates metastasis to other organs and results in a poor prognosis in patients $(29,30)$. In the present study, the migration assay test was used to investigate the potential impact of upregulation of ZBTB7A on GC cell migration (Fig. 5). The migratory rate of OE group was $0.17 \pm 0.02$, in contrast with $0.28 \pm 0.03$ of $\mathrm{NC}$ group and $0.24 \pm 0.03$ of CON group at $8 \mathrm{~h}$ (Fig. 5B). Following $24 \mathrm{~h}$, the migratory rate of the $\mathrm{CON}, \mathrm{NC}$ and $\mathrm{OE}$ groups were $0.46 \pm 0.04,0.49 \pm 0.03$ and $0.35 \pm 0.02$, respectively (Fig. $5 \mathrm{~B}$ ). Another three repeats were conducted independently. Statistical analysis demonstrated that the migratory rate of cells in the OE group at 8 and $24 \mathrm{~h}$ time points were significantly decreased compared with the CON group and NC group (Fig. 5B), suggesting that overexpression of ZBTB7A repressed GC cell migration.

\section{Discussion}

TCGA provides a large amount of data, serving an important resource for the field of cancer research (31). Previously, a number of studies have demonstrated that the ZBTB7A gene locus exhibited frequent chromosomal deletions in a number of different types of human cancer (18-20). In the present study, data mining of ZBTB7A in TCGA gastric adenocarcinoma dataset was performed. It was demonstrated that frequent loss of the ZBTB7A gene also occurred in gastric adenocarcinoma, with $37.17 \%$ gene loss compared with $56.56 \%$ normal gene status. Deletion of the ZBTB7A gene resulted in a decreased in mRNA expression of ZBTB7A. In addition, survival analysis demonstrated that downregulation of ZBTB7A was associated with a poor prognosis in patients with gastric adenocarcinoma.

ZBTB7A is a member of the POK transcription factor family (9-11), which was previously known as a proto-oncogene that acts by suppressing the transcription of an ARF of a tumor suppressor (11). Previously, ZBTB7A has also been reported to be novel proto-oncogene in different types of cancer, including breast cancer, NSCL, lymphoma and ovarian cancer (13-17). However, the frequent chromosomal deletion of the ZBTB7A gene locus in numerous types of human cancer (18-20) contradicts its proto-oncogenic role. Previous studies indicate that ZBTB7A works as a tumor suppressor in melanoma (20), PTEN-loss background prostate cancer (21) and colonic cancer (22). In the present study, it was identified that ZBTB7A overexpression induced an abnormal proportion of cells to be in the $S$ phase; however, this had no impact on cell proliferation. Furthermore, in the gain-of-function assay, ZBTB7A promoted cell apoptosis and repressed cell migration in the SGC-7901 cell line. The present study indicates that ZBTB7A functions as a tumor suppressor in GC SGC-7901 cell line.

Recently it's been reported that downregulation of ZBTB7A by siRNA, repressed the migratory ability of GC cells without an impact on cell proliferation and apoptosis (23), without presenting detailed mechanisms. In the present study, upregulation of ZBTB7A also suppressed migratory ability. This phenomenon deserves further investigation. It was also identified that gain-of-function of ZBTB7A in the SGC-7901 cell line promotes apoptosis; however, there was no impact on apoptosis when downregulation of ZBTB7A was investigated (23). The abnormal cell cycle $S$ phase accumulation induced by ZBTB7A overexpression indicated that ZBTB7A may promote the transcription of different target genes depending on whether it is up- or downregulated. This implies that the function of ZBTB7A in GC may be background status dependent.

It was reported that ZBTB7A acted as a proto-oncogene in certain contexts but also exhibited tumor suppressive activity in PTEN deficient tumors (29). This suggested that ZBTB7A may possess onco-suppressive activity in PTEN-deleted gastric adenocarcinoma as well as in PTEN-deficit prostate cancer (29). This demonstrates that the role of ZBTB7A in GC may be PTEN deficit associated context-dependent.

In conclusion, the present study identified a novel genetic event associated with gastric adenocarcinoma, the frequent loss of ZBTB7A gene. Deletion of ZBTB7A was associated with a poor prognosis in patients with GC. Gain-of-function of ZBTB7A demonstrated tumor suppressive-like activity, including inducing cell cycle arrest at $\mathrm{S}$ phase, promoting apoptosis and repressing cell migration in a GC cell line. The present study indicated that ZBTB7A functioned as tumor 
suppressor in GC cells, which may offer therapeutic or prognostic implications for patients with GC in future.

\section{Acknowledgements}

The authors of the present study would like to thank Shanghai GeneChem Co., Ltd. (Shanghai, China) for their technical assistance. In addition, the authors would like to thank the core lab of the Haikou City Hospital for offering numerous suggestions. The authors also would like to thank Dr Hongman Wu (Haikou Municipal People's Hospital) for his advice.

\section{References}

1. Ferlay J, Soerjomataram I, Dikshit R, Eser S, Mathers C, Rebelo M, Parkin DM, Forman D and Bray F: Cancer incidence and mortality worldwide: Sources, methods and major patterns in GLOBOCAN 2012. Int J Cancer 136: E359-E386, 2015.

2. Van Cutsem E, Sagaert X, Topal B, Haustermans K and Prenen H: Gastric cancer. Lancet 388: 2654-2664, 2016.

3. Soerjomataram I, Lortet-Tieulent J, Parkin DM, Ferlay J, Mathers C, Forman D and Bray F: Global burden of cancer in 2008: A systematic analysis of disability-adjusted life-years in 12 world regions. Lancet 380: 1840-1850, 2012.

4. Tan P and Yeoh KG: Genetics and molecular pathogenesis of gastric adenocarcinoma. Gastroenterology 149: 1153-1162.e3, 2015.

5. Cancer Genome Atlas Research Network: Comprehensive molecular characterization of gastric adenocarcinoma. Nature 513: 202-209, 2014.

6. Liu CJ, Prazak L, Fajardo M, Yu S, Tyagi N and Di Cesare PE: Leukemia/lymphoma-related factor, a POZ domain-containing transcriptional repressor, interacts with histone deacetylase-1 and inhibits cartilage oligomeric matrix protein gene expression and chondrogenesis. J Biol Chem 279: 47081-47091, 2004.

7. Pessler F, Pendergrast PS and Hernandez N: Purification and characterization of FBI-1, a cellular factor that binds to the human immunodeficiency virus type 1 inducer of short transcripts. Mo Cell Biol 17: 3786-3798, 1997.

8. Kukita A, Kukita T, Ouchida M, Maeda H, Yatsuki H and Kohashi O: Osteoclast-derived zinc finger (OCZF) protein with $\mathrm{POZ}$ domain, a possible transcriptional repressor, is involved in osteoclastogenesis. Blood 94: 1987-1997, 1999.

9. Davies JM, Hawe N, Kabarowski J, Huang QH, Zhu J, Brand NJ, Leprince D, Dhordain P, Cook M, Morriss-Kay G and Zelent A: Novel BTB/POZ domain zinc-finger protein, LRF, is a potential target of the LAZ-3/BCL-6 oncogene. Oncogene 18: 365-375, 1999.

10. Apostolopoulou K, Pateras IS, Evangelou K, Tsantoulis PK, Liontos M, Kittas C, Tiniakos DG, Kotsinas A, Cordon-Cardo C and Gorgoulis VG: Gene amplification is a relatively frequent event leading to ZBTB7A (Pokemon) overexpression in non-small cell lung cancer. J Pathol 213: 294-302, 2007.

11. Maeda T, Hobbs RM, Merghoub T, Guernah I, Zelent A, Cordon-Cardo C, Teruya-Feldstein J and Pandolfi PP: Role of the proto-oncogene Pokemon in cellular transformation and ARF repression. Nature 433: 278-285, 2005.

12. Costoya JA: Functional analysis of the role of POK transcriptional repressors. Brief Funct Genomic Proteomic 6: 8-18, 2007.

13. Jiang L, Siu MK, Wong OG, Tam KF, Lam EW, Ngan HY, Le XF, Wong ES, Chan HY and Cheung AN: Overexpression of proto-oncogene FBI-1 activates membrane type 1-matrix metalloproteinase in association with adverse outcome in ovarian cancers. Mol Cancer 9: 318, 2010.
14. Aggarwal A, Hunter WJ III, Aggarwal H, Silva ED Davey MS, Murphy RF and Agrawal DK: Expression of leukemia/lymphoma-related factor (LRF/POKEMON) in human breast carcinoma and other cancers. Exp Mol Pathol 89: 140-148, 2010.

15. Qu H, Qu D, Chen F, Zhang Z, Liu B and Liu H: ZBTB7 overexpression contributes to malignancy in breast cancer. Cancer Invest 28: 672-678, 2010.

16. Vredeveld LC, Rowland BD, Douma S, Bernards R and Peeper DS: Functional identification of LRF as an oncogene that bypasses RASV12-induced senescence via upregulation of CYCLIN E. Carcinogenesis 31: 201-207, 2010.

17. Zhao ZH, Wang SF, Yu L, Wang J, Chang H, Yan WL, Zhang J and $\mathrm{Fu} \mathrm{K}$ : Overexpression of Pokemon in non-small cell lung cancer and foreshowing tumor biological behavior as well as clinical results. Lung Cancer 62: 113-119, 2008.

18. Beroukhim R, Mermel CH, Porter D, Wei G, Raychaudhuri S, Donovan J, Barretina J, Boehm JS, Dobson J, Urashima M, et al: The landscape of somatic copy-number alteration across human cancers. Nature 463: 899-905, 2010

19. Zack TI, Schumacher SE, Carter SL, Cherniack AD, Saksena G, Tabak B, Lawrence MS, Zhsng CZ, Wala J, Mermel CH, et al: Pan-cancer patterns of somatic copy number alteration. Nat Genet 45: 1134-1140, 2013.

20. Liu XS, Genet MD, Haines JE, Mehanna EK, Wu S, Chen HI, Chen Y, Qureshi AA, Han J, Chen X, et al: ZBTB7A Suppresses melanoma metastasis by transcriptionally repressing MCAM. Mol Cancer Res 13: 1206-1217, 2015.

21. Wang G, Lunardi A, Zhang J, Chen Z, Ala U, Webster KA, Tay Y, Gonzalez-Billalabeitia E, Egia A, Shaffer DR, et al: Zbtb7a suppresses prostate cancer through repression of a Sox9-dependent pathway for cellular senescence bypass and tumor invasion. Nat Genet 45: 739-746, 2013.

22. Liu XS, Haines JE, Mehanna EK, Genet MD, Ben-Sahra I, Asara JM, Manning BD and Yuan ZM: ZBTB7A acts as a tumor suppressor through the transcriptional repression of glycolysis. Genes Dev 28: 1917-1928, 2014.

23. Shi DB, Wang YW, Xing AY, Gao JW, Zhang H, Guo XY and Gao P: C/EBP $\alpha$-induced miR-100 expression suppresses tumor metastasis and growth by targeting ZBTB7A in gastric cancer. Cancer Lett 369: 376-385, 2015.

24. Gao J, Aksoy BA, Dogrusoz U, Dresdner G, Gross B, Sumer SO, Sun Y, Jacobsen A, Sinha R, Larsson E, et al: Integrative analysis of complex cancer genomics and clinical profiles using the cBioPortal. Sci Signal 6: pl1, 2013.

25. Cerami E, Gao J, Dogrusoz U, Gross BE, Sumer SO, Aksoy BA, Jacobsen A, Byrne CJ, Heuer ML, Larsson E, et al: The cBio cancer genomics portal: An open platform for exploring multidimensional cancer genomics data. Cancer Discov 2: 401-404, 2012.

26. Livak KJ and Schmittgen TD: Analysis of relative gene expression data using real-time quantitative PCR and the 2(-Delta Delta C(T)) method. Methods 25: 402-408, 2001.

27. Bengtsson M, Hemberg M, Rorsman P and Ståhlberg A: Quantification of mRNA in single cells and modelling of RT-qPCR induced noise. BMC Mol Biol 9: 63, 2008.

28. Kubista M, Andrade JM, Bengtsson M, Forootan A, Jonák J, Lind K, Sindelka R, Sjöback R, Sjögreen B, Strömbom L, et al: The real-time polymerase chain reaction. Mol Aspects Med 27: 95-125, 2006

29. Wang SM, Tie J, Wang WL, Hu SJ, Yin JP, Yi XF, Tian ZH, Zhang XY, Li MB, Li ZS, et al: POU2F2-oriented network promotes human gastric cancer metastasis. Gut 65: 1427-1438, 2016.

30. Zhang ZY and Ge HY: Micrometastasis in gastric cancer. Cancer Lett 336: 34-45, 2013

31. Garraway LA and Lander ES: Lessons from the cancer genome. Cell 153: 17-37, 2013. 